\title{
Towards NEMS Fluid Sensors Based on Suspended Nanomaterials
}

\author{
Luis A. Jauregui ${ }^{1,3}$, Hao $\mathrm{Lu}^{4,5}$, Jun Lou ${ }^{4,5}$, Yong P. Chen ${ }^{2,3,1}$
}

1. School of Electrical and Computer Engineering, Purdue University, West Lafayette, IN, USA. 2. Department of Physics, Purdue University, West Lafayette, IN, USA.

3. Birck Nanotechnology Center, Purdue University, West Lafayette, IN, USA.

4. Department of Mechanical Engineering \& Materials Science, Rice University, Houston, TX, USA.

5. Richard E. Smalley Institute for Nanoscale Science and Technology, Rice University, Houston, TX, USA.

\begin{abstract}
:
Nanoelectromechanical systems (NEMS) based on nanomaterials, especially NEMS resonators made of suspended nanowires, nanotubes or nanosheets have emerged as promising devices for many sensing applications. However, operation of such NEMS resonators in liquids is usually difficult due to strong viscous damping. Here we present our progress in developing NEMS devices based on suspended nanomaterials that can operate in liquids. We present our measurements performed on suspended metallic nanowires driven by AC currents in a magnetic field in liquids.
\end{abstract}

\section{INTRODUCTION:}

Nanoelectromechanical systems based on nanomaterials promise extraordinary sensitivity in the detection of displacement, mass, force and charge. Adsorption of molecules is measured by changes on the resonant frequency. However mostly they have been operated under vacuum conditions. Air or fluidic environments [1] introduces damping effects which degrades the quality factor of the resonators, reducing its sensitivity, making almost impossible to measure small masses under fluidic environments. Few experiments have been realized to analyze the behavior of nanoelectromechanical systems in fluids such as air [2] and MEMS devices in liquid environments [3]. Motivated by this we explore the discrimination of different liquids with different viscosity by the time dependant measurements of the mechanical motions (eg., driven or damped oscillations). 


\section{EXPERIMENTAL DETAILS:}

We use magnetomotive driving forces [4] in order to make our devices oscillate and use electrical read-outs to get their response under different magnetic fields. We create suspended gold nanowires on 300nm silicon oxide substrates (with etched trenches of 300nm), with Au/Ti $(100 \mathrm{~nm} / 5 \mathrm{~nm})$ electrodes defined by e-Beam lithography.
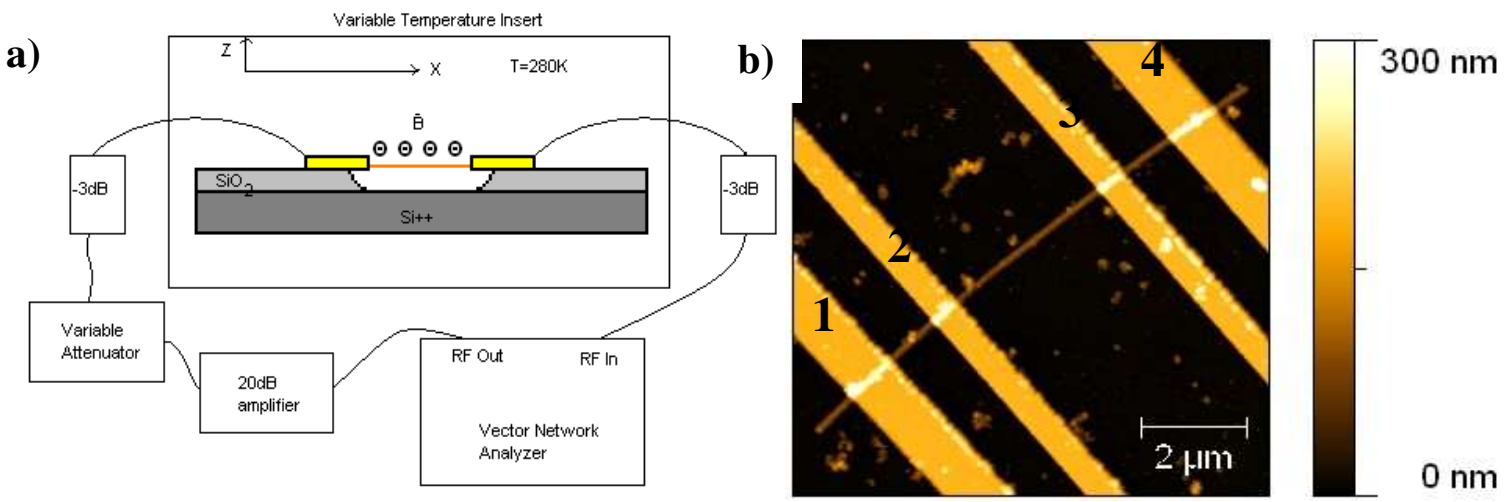

Figure 1. a) magnetomotive experimental layout, where the magnetic field is pointing out the plane. b)Nanowire clamped by gold electrodes, defined by eBeam lithography, before suspension process.

After that, we characterize by atomic force microscope to get the geometrical factors of the suspended nanowires. The device is wire bonded and mounted in the cavity of a liquid-tight ceramic package, and then it is inserted in a variable temperature insert (VTI) and maintained at a temperature of $\sim 300 \mathrm{~K}$. The VTI is equipped with a superconducting magnet that can provide a maximum magnetic field of $7 \mathrm{~T}$ (while the sample is in an isolated various temperature/pressure chamber) in order to make the nanowire resonate. For that purpose, an $\mathrm{AC}$ current is applied on the nanowire by a $\mathrm{RF} /$ microwave vector network analyzer as shown in the schematic in figure 1a. The current is applied between electrodes 1 and 2 as shown in figure 1b. After the nanowires are characterized, we introduce silicon oil of 10cSs in the cavity and seal it by a glass lid. Finally, we measure the resonance of the device in the liquid. We compare the behavior of the devices with and without liquids environments. 

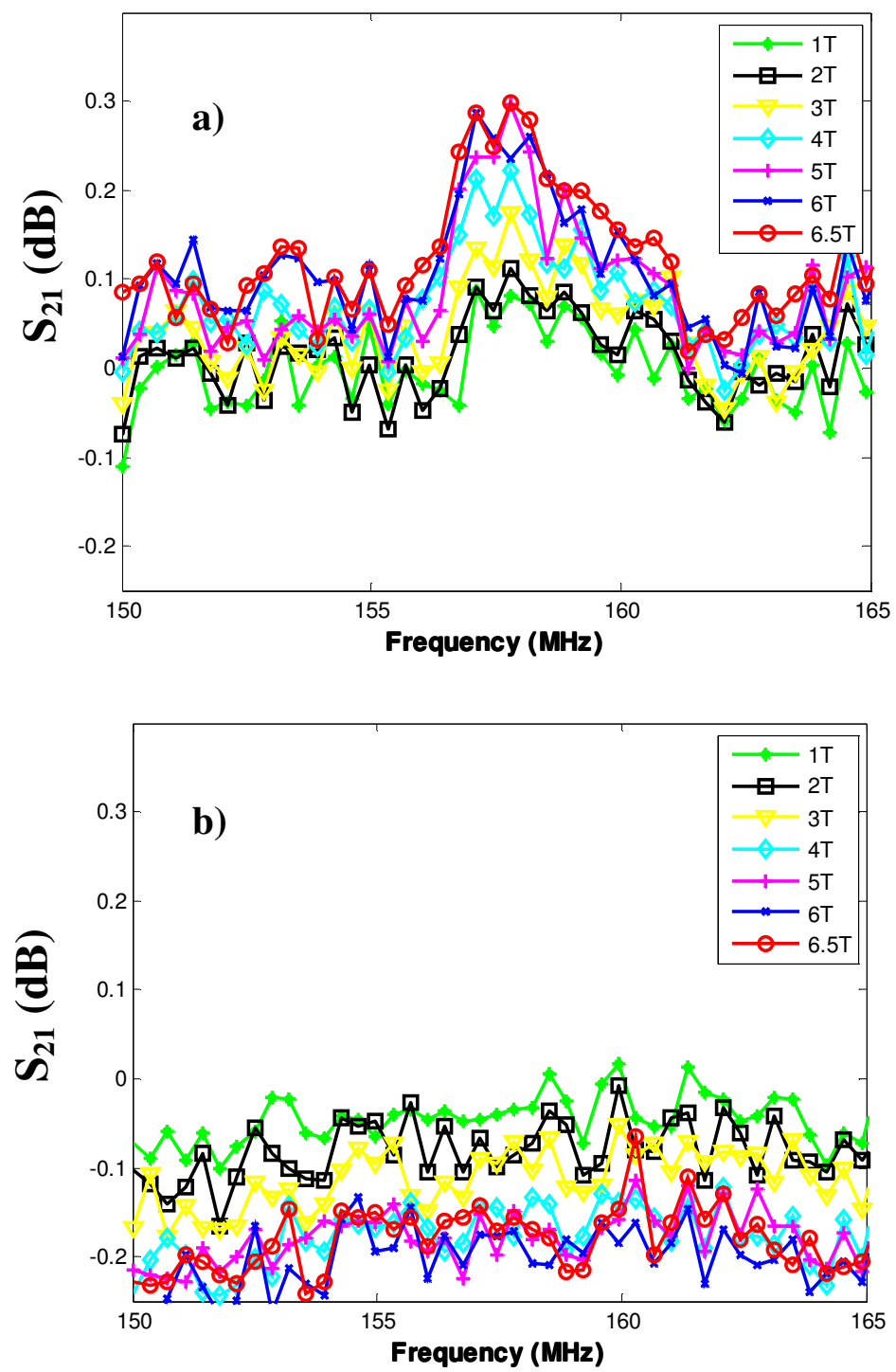

Figure 2. a) Gold nanowire first resonant peak for different applied magnetic fields in vacuum, b) under silicone oil 10cSt. 

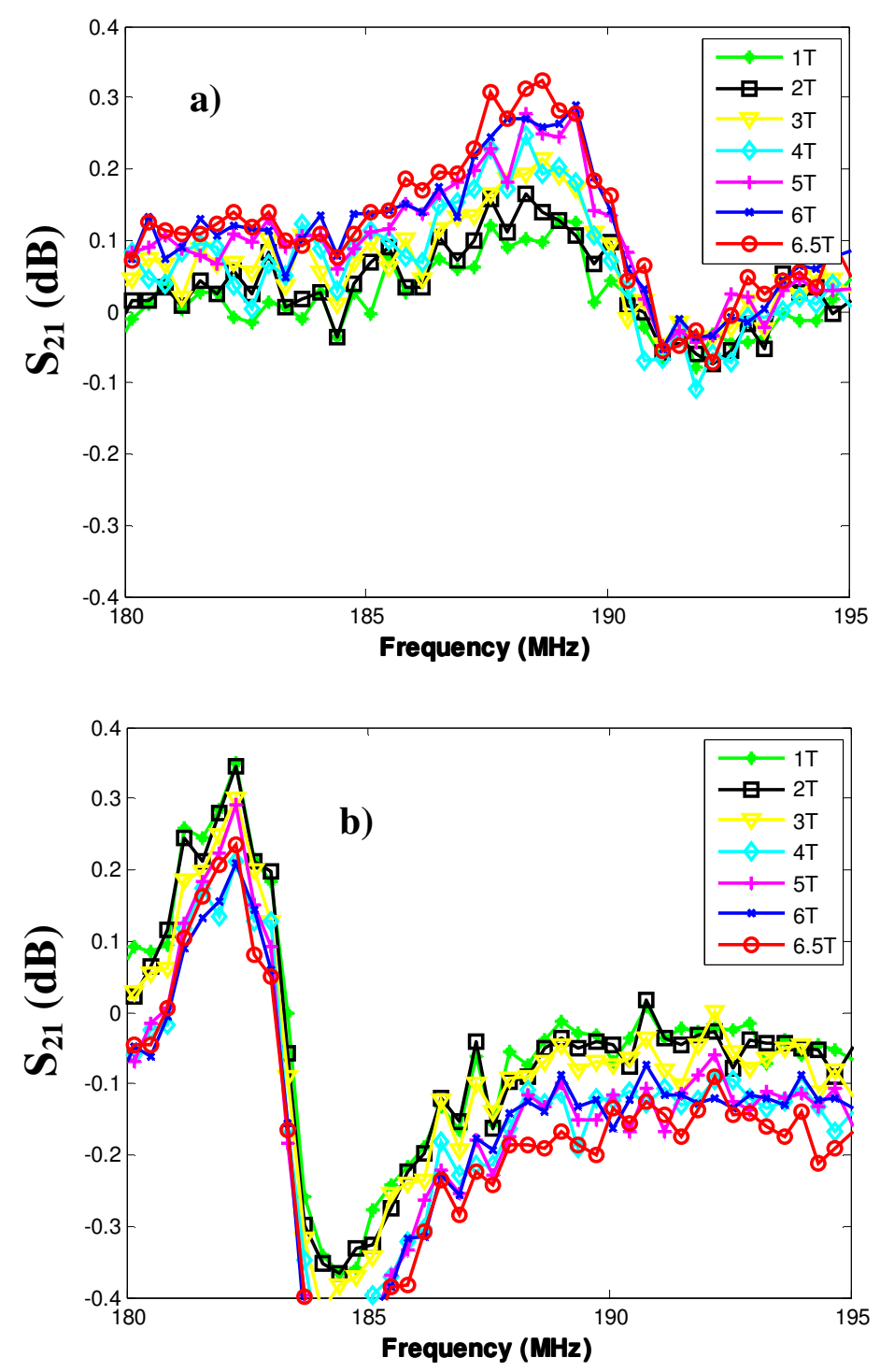

Figure 3. a) Gold nanowire second resonant peak for different applied magnetic fields in vacuum.b) under silicone oil 10cSt.

\section{RESULTS AND CONCLUSION:}

Figures $2 \mathrm{a}$ and $3 \mathrm{a}$ show the measured S21 spectra, containing what we refer to as the first and second resonant peak respectively of a suspended gold nanowire device in vacuum, under different applied magnetic fields. Figure $2 \mathrm{~b}$ and $3 \mathrm{~b}$ show what happen to the first and second resonant peaks of the same gold nanowire when placed in silicone oil of 10cSt. Our results show the two peaks (in vacuum) in the range of 5-200 MHz, which could be associated with the mechanical resonances of the nanowire if we take into account of a negative residual tension that can give more than one solutions to the Euler equation. With this interpretation, the small shifts 
of the resonant frequency in liquid for the $2^{\text {nd }}$ peak could be due to added mass that may change the roots of this equation, shifting the resonant peak [5]. While the first peak is completely damped in liquid environment, the second peak is not and its amplitude of oscillations increases with increasing applied magnetic field. The observed resonances and the mechanisms that the liquid environment may attenuate or amplify or change the shape of these resonances are under further investigation. We may perform sensing in liquids based on the time-domain information (such as damping time) in addition to any frequency shifts.

\section{ACKNOWLEDGEMENT:}

This research is supported by NSF Grant ECCS 0702766.

\section{REFERENCES:}

1. Sazonova, V., Yaish, Y., Ustunel, H., Roundy, D., Arias, T.A., and McEuen, P.L.: 'A tunable carbon nanotube electromechanical oscillator', Nature, 2004, 431, (7006), pp. 284-287

2. Sekaric, L., Zalalutdinov, M., Bhiladvala, R.B., Zehnder, A.T., Parpia, J.M., and Craighead, H.G.: 'Operation of nanomechanical resonant structures in air', Applied Physics Letters, 2002, 81, (14), pp. 2641-2643

3. Etchart, I., Chen, H., Dryden, P., Jundt, J., Harrison, C., Hsu, K., Marty, F., and Mercier, B.: 'MEMS sensors for density-viscosity sensing in a low-flow microfluidic environment', Sensors and Actuators a-Physical, 2008, 141, (2), pp. 266-275

4. Husain, A., Hone, J., Postma, H.W.C., Huang, X.M.H., Drake, T., Barbic, M., Scherer, A., and Roukes, M.L.: 'Nanowire-based very-high-frequency electromechanical resonator', Applied Physics Letters, 2003, 83, (6), pp. 1240-1242

5. Seoanez, C., Guinea, F., and Castro, A.H.: 'Dissipation in graphene and nanotube resonators', Physical Review B, 2007, 76, (12), pp. 8 